\title{
VillanueVa Flores, Rocío (2009). Derecho a la salud, perspectiva de género y multiculturalismo. Lima: Palestra Editores, 288 pp.
}

El derecho a la salud es un tema poco tratado en nuestro país, lo que evidencia a mi juicio una falencia en el tratamiento doctrinario que se haga de los derechos sociales reconocidos en nuestra Constitución. Sin embargo, hoy en día es posible observar algunos esfuerzos en nuestra doctrina ${ }^{1}$ que buscan dotar de contenido a este derecho, siguiendo tendencias internacionales - principalmente la contenida en las Observaciones Generales emanadas de los distintos Comités de Naciones Unidas que supervisan la aplicación de diferentes pactos internacionales. Otro ejemplo de ello son las sentencias emanadas de la Corte de Apelaciones de Santiago, de noviembre de 2009, las que acogen sendos recursos de protección en contra del Servicio de Salud Metropolitano y en contra de FONASA, obligando al Estado a solventar los gastos médicos que requiere el tratamiento de dos mujeres con enfermedades terminales ${ }^{2}$, otorgando protección efectiva a un derecho de naturaleza social, basándose en el principio de supremacía constitucional y en las obligaciones que emanan de la suscripción del Pacto Internacional de Derechos Económicos, Sociales y Culturales.

Estas observaciones son las que me motivan a presentar el libro de la Dra. Villanueva Flores, toda vez que esta monografía es un ejemplo de especificación de un tema que en sí mismo es muy complejo, pero que la autora logra abordar ilustrando, a través de casos y sentencias, el tratamiento de las diferentes aristas a que da lugar el ejercicio y protección del derecho a la salud con fenómenos tales como la violencia de género, el respeto a los derechos reproductivos de la mujer y la adaptación de las tradiciones y de la cultura indígena a las exigencias mínimas de respeto a la dignidad de las personas, individualmente consideradas, que nos impone el funcionamiento de los Estados constitucionales de Derecho actuales, lo que supone analizar los problemas que plantea el multiculturalismo y el derecho a la diversidad étnica y cultural.

El hilo conductor que origina este estudio es la perspectiva de género, la que supone abordar el análisis del objeto de estudio tomando en consideración tres elementos: el concepto de género; las relaciones de género; y el sistema de género y, a partir de ellos, dejar en evidencia la necesidad de introducir ciertos cambios al sistema jurídico, a fin de hacer plenamente efectivo el derecho a la salud.

\footnotetext{
1 Por ejemplo, Noguerra Alcalá, Humberto (2009). Derechos fundamentales y garantias constitucionales, tomo III. Santiago: Librotecnia, pp. 105-297.

2 Corte de Apelaciones de Santiago. Sentencia de 24 de noviembre de 2009, Rol 7766-2009.

"Patricia Vásquez Ibarra con Fonasa".
} 
Los estudios analíticos de género, que datan de la década del '70 en adelante, nos describen la construcción de la identidad sexual de un infante, ya no derivado de su sexo genético o biológico, sino de la designación de género que se le hace al nacer. Ese proceso, que hoy gracias a los avances médicos es previo incluso al nacimiento, desencadena un conjunto de actitudes polarizadas, de respuestas humanas diferentes y diferenciadas que acompañan al niño o niña hasta la muerte. Ya al ańo y medio de vida los bebés experimentan un sentimiento íntimo y confuso de ser hombre o mujer, estableciéndose el núcleo de su identidad sexual. De ello podemos concluir en primer lugar que los sujetos han sido sexuados por la interacción con sus semejantes, por su familia nuclear en primer lugar, de un modo irreversible; y que la construcción del psiquismo femenino o masculino es el resultado de una historia individual, familiar y social.

Por tanto, es la historia y la biografía personal la que construye, a partir de nuestra dotación genética, la subjetividad de cada individuo. Desde esta perspectiva, la fisiología no influye en la construcción de ciertos valores sobre los cuerpos de las mujeres o de los hombres. Además de lo biológico, debieran considerarse los factores psicológicos, ontológicos, culturales, históricos, sociales y económicos. Es la sociedad, a través de la cultura, la que determina las características "femeninas" y "masculinas"; por tanto, estas no son algo natural, sino que, por el contrario, son un producto cultural, por ende, esas características se reproducen en las instituciones educativas, legales, políticas, religiosas, etc., a través de símbolos, disposiciones legales, prácticas culturales y valores sociales.

Desde esta perspectiva -señala Villanueva Flores- la inclusión del género como categoría conceptual está asociada a las demandas de las mujeres para revelar y superar la desigualdad social, puesto que existen distintos tipos de recursos a los que las mujeres tienen menos acceso y control que los hombres, de entre ellos, el acceso a los servicios de salud y la información sobre la propia salud. De allí que la autora se proponga demostrar la necesidad de que tanto la legislación, las políticas sociales impulsadas por el Estado, así como la jurisprudencia deba considerar necesariamente el enfoque de género cuando se trate de la protección del derecho a la salud, puesto que estas fuentes formales y materiales del Derecho también son parte del sistema de género.

El primer punto de análisis que se aborda es la violencia de género y la protección de los derechos reproductivos de las mujeres, el que abarca el ámbito familiar, el medio social y el medio laboral. En todos estos campos, a pesar de la introducción de una serie de cambios legislativos en países de la región, la violencia de género sigue produciéndose, puesto que esta es el resultado de la actuación de factores socioculturales. Y al ser considerada por la OMS como un ejemplo de violación a derechos humanos, este problema de naturaleza cultural se convierte en un problema de salud pública y en un obstáculo al desarrollo social y democrático de los 
países, puesto que esta tiene costos directos, que están referidos a la pérdida de vidas, al valor de los bienes y servicios empleados en el tratamiento y prevención de la violencia, incluidos los gastos judiciales, en salud o servicios sociales asumidos por la propia víctima o por la comunidad; tiene costos indirectos, entre los que se cuentan las tasas de aborto, las pérdidas de productividad económica y las que derivan de la falta de participación de las mujeres en los procesos de desarrollo social, político y económico; y finalmente, genera también costos intangibles, como es la transmisión intergeneracional de la violencia.

Aquí la autora acompaña y analiza una serie de sentencias judiciales de la Corte Interamericana de Derechos Humanos, tales como la sentencia de 25 de noviembre de $2006^{3}$, la que es especialmente importante ya que junto con ser la primera resolución que aborda directamente el problema de la violencia de género, es una sentencia que además define este concepto como actos dirigidos o planificados hacia las mujeres, que resultan agravados por su condición de tales o que las afectan de manera diferente o en mayor proporción $n^{4}$, y además se pronuncia sobre la aplicación de la Convención de Belem do Pará y las obligaciones que de ella derivan para los Estados, así como también advierte la especial situación de las mujeres internas en un presidio y que se encuentren embarazadas (tal como sucedió en el Penal Miguel Castro Castro de Lima). Junto a estas cuestiones, la sentencia aborda el tema del desnudo forzado como forma de violencia sexual, la inspección vaginal como forma de violación sexual (y que configura un ejemplo de tortura), las condiciones de la detención; y finalmente, aborda también la relación de los hechos que originaron la causa con los delitos de lesa humanidad.

En el segundo punto de análisis, los derechos reproductivos, la autora recurre a una serie de instrumentos internacionales, tales como la Convención para la Eliminación de todas las formas de discriminación en contra de la mujer, el Programa de Acción de El Cairo, la Plataforma de Acción de Beijing, algunas resoluciones de la Comisión de Derechos Humanos de Naciones Unidas, a fin de determinar el concepto de salud reproductiva y las obligaciones que este impone a los Estados, para luego limitarse al análisis de la faceta negativa que poseen estos derechos,

3 Corte Interamericana de Derechos Humanos. Sentencia de 25 de noviembre de 2006, caso Penal Miguel Castro Castro versus Perú.

4 Corte Interamericana de Derechos Humanos. Sentencia de 28 de enero de 2009, caso Ríos y otros versus Venezuela, y sentencia de 28 de enero de 2008, caso Perozo y otros versus Venezuela.

5 Como por ejemplo el Comité de Derechos Humanos; el Comité de Derechos Económicos sociales y culturales; el Comité sobre los Derechos del Nińo y el Comité de la CEDAW. Entre las resoluciones más importantes destaca la Observación General No 28 del Comité de Derechos Humanos, las Recomendaciónes Generales No 19, 21 y 24 del Comité de la CEDAW y la Observación General No 4 del Comité de los Derechos del Niño. 
es decir, vinculados con la facultad de las mujeres de no reproducirse a través de la utilización de la anticoncepción oral de emergencia (AOE), abarcando en específico la regulación y tratamiento jurisprudencial que este fármaco ha tenido en esta región del planeta, ya que la presentación en diferentes países de Latinoamérica de varias demandas contra la $\mathrm{AOE}^{6}$, pusieron de manifiesto - a juicio de la autora- la vinculación entre los derechos reproductivos, la libertad individual y el derecho de las mujeres a no ser discriminadas.

Y trata el tema desde una perspectiva de género porque estima que si bien hombres y mujeres son titulares de derechos reproductivos (recordemos que la titularidad de los derechos es universal), seńala que el ejercicio de estos derechos cobra una importancia especial tratándose de ellas, pues son quienes asumen el costo biológico y gran parte del costo social de la reproducción.

Finalmente, el tercer punto de análisis son los posibles conflictos que en el ámbito de la salud se pueden presentar entre los derechos de las comunidades indígenas y los derechos individuales de sus integrantes, ya que el respeto de los derechos humanos es el límite del derecho de los pueblos indígenas y tribales a conservar sus costumbres e instituciones. En este sentido, cabe destacar que en América Latina existen unos 642 pueblos indígenas, lo que equivale alrededor de 30 millones de personas.

En esta ocasión, el marco normativo internacional que sirve de base teórica para las reflexiones que nos presenta la autora son el Convenio $\mathrm{N}^{\circ}$ 169 de la OIT, la Declaración de las Naciones Unidas sobre los derechos de los pueblos indígenas y la Carta Andina para la Promoción y Protección de los Derechos Humanos, y, en el ámbito jurisprudencial, recurre a una serie de sentencias de la Corte Interamericana de Derechos Humanos, como de tribunales y cortes constitucionales.

Resulta interesante este tema, ya desde sus inicios, puesto que los derechos colectivos que se reconocen a los grupos o comunidades indígenas, que por lo general son definidos como derechos culturales ${ }^{7}$, escapan completamente a la nomenclatura clásica, de corte liberal e individualista, que hasta hace unos pocos ańos primaba para el análisis y la discusión sobre derechos humanos o derechos fundamentales. Hoy en día la cuestión pasa por considerar varios temas, no solo aquellos relacionados con el ejercicio de los derechos colectivos o su protección jurisdiccional, sino que tam-

\footnotetext{
6. Que específicamente buscaban la prohibición judicial de la comercialización y distribución gratuita del fármaco. Este tema es tratado en extenso por la Dra. Villanueva en su libro de 2008 La anticoncepción oral de emergencia. El debate legal en América Latina. San José: IIDH.

7 Que incluyen el derecho al acceso a la cultura, el derecho a la participación en la vida cultural y a su disfrute, y los derechos de protección de las creaciones científicas y artísticas. DE Lucas, Pablo (2003). "¿Qué quiere decir tener derecho a la cultura?", en ABRAmovich, Víctor y Añón, María José: Derechos sociales. Instrucciones de uso. México: Fontamara, p. 303.
} 
bién es posible cuestionar -tal como lo hace Comanducci ${ }^{8}$ - quién determina o decide cuál es la tradición o cultura válida para una comunidad determinada.

Es por ello que la sistematización y examen de varias sentencias internacionales ${ }^{9} \mathrm{y}$ algunas nacionales ${ }^{10}$ es un gran aporte en este sentido, ya que ayuda a dilucidar los criterios interpretativos con que contamos para delinear posibles conflictos de derechos, y algunos de ellos ya han originado situaciones difíciles en nuestro país, los que dicen relación con una necesidad de reivindicar la defensa de determinados derechos colectivos, tales como las subvenciones públicas para la difusión de la propia lengua, el reconocimiento de prácticas jurídicas, la propiedad colectiva, la representación ante instancias políticas, entre otras.

Nuevamente, la autora ocupa la perspectiva de género para su análisis, principalmente porque considera que las mujeres indígenas son víctimas de una triple discriminación: por ser mujeres, indígenas y pobres, lo que ha originado una serie de amenazas a sus derechos fundamentales individuales, fundadas en tradiciones culturales, tales como el matrimonio por captura, que origina delitos de secuestro y violación sexual, los matrimonios forzados a menores de edad, mutilación femenina, ciertos ritos y costumbres matrimoniales, la denegación del acceso a la propiedad, entre otras.

La posibilidad de empoderar a las mujeres de nuestro continente es un tema que contiene aristas éticas, políticas y jurídicas. Ya en el Foro Social Mundial de 2005, realizado en Porto Alegre, Brasil, se alude al hecho de que la salud de la mujer solo se la relaciona con la maternidad y el cuidado de otros, pero no son discutidos sus derechos sobre su propio cuerpo, ni se reconoce tampoco el impacto que la pobreza o las condiciones de origen étnico tienen sobre la salud, es por ello que pienso que monografías como estas son necesarias para dilucidar y profundizar aún más el debate en temas tan sensibles como el ejercicio del derecho a la salud, en donde al parecer nos queda mucho camino por recorrer.

Comanducci, Paolo (2001). "Derechos humanos y minorías: un acercamiento analítico neoilustrado". En Carbonell, Miguel / Cruz Pacero, Juan A. / VÁsquez, Rodolfo: Derechos sociales y derechos de las minorias, México: UNAM, Ed. Porrúa.

9 Todas de la Corte Interamericana de Derechos Humanos: caso Comunidad Mayagna (Sumo) Awas Tingni vs. Nicaragua, de 31 de agosto de 2001; caso Comunidad Moiwana vs. Suriname, de 15 de junio de 2005; caso Comunidad Indigena Yakye Axa vs. Paraguay, de 17 de junio de 2005; caso Comunidad Indigena Sawhoyamaxa vs. Paraguay, de 29 de marzo de 2006 y caso Pueblo de Saramaka vs. Surinam, de 28 de noviembre de 2007.

10 Utiliza sentencias de la Corte Constitucional colombiana, del Tribunal Constitucional del Perú y del antiguo Tribunal Constitucional de Bolivia que se rigió por la Ley No 2631 de 2004 . 
Como señala Sherwin ${ }^{11}$, para que las mujeres logren total subjetividad y sean consideradas sujetos de derechos plenos, y por ende personas, con todo lo que ello implica, es necesario que estén libres de distintas formas de subordinación, de violencia y de la pobreza.

CAROLina SALAS SALAZAR*

11 Sherwin, Susan (1998). The politics of women's Health. Filadelfia: Temple University Press. Abogada. Doctora en Derecho Constitucional por la Universidad de Castilla - La Mancha (España). Profesora de Derecho Constitucional de la Universidad Católica del Norte, sede Coquimbo. Correo electrónico: csalas@ucn.cl 